\title{
Spherical-harmonics expansion method for density-matrix simulations of quantum electron dynamics in continuum states
}

\author{
AUTHOR(S): \\ Kitamura, Hikaru
}

\section{CITATION:}

Kitamura, Hikaru. Spherical-harmonics expansion method for density-matrix simulations of quantum electron dynamics in continuum states. International Journal of Quantum Chemistry 2015, 115(22): 1587-1596

\section{ISSUE DATE:}

2015-11-15

URL:

http://hdl.handle.net/2433/203165

\section{RIGHT:}

This is the peer reviewed version of the following article: Hikaru Kitamura. Spherical-harmonics expansion method for density-matrix simulations of quantum electron dynamics in continuum states. nternational Journal of Quantum Chemistry Volume 115, Issue 22, pages 1587?1596, which has been published in final form at

http://dx.doi.org/10.1002/qua.24988. This article may be used for non-commercial purposes in accordance with Wiley Terms and Conditions for Self-Archiving.; The full-text file will be made open to the public on 10 August 2016 in

accordance with publisher's 'Terms and Conditions for Self-Archiving'; この論文は出版社版でありません。引用の際に は出版社版をご確認ご利用ください。; This is not the published version. Please cite only the published version. 


\title{
Spherical-Harmonics Expansion Method for Density-Matrix Simulations of Quantum Electron Dynamics in Continuum States
}

\section{Hikaru Kitamura ${ }^{1}$}

\author{
${ }^{1}$ Hikaru Kitamura \\ Department of Physics, Kyoto University, Sakyo-ku, Kyoto 606-8502, Japan \\ E-mail: kitamura@scphys.kyoto-u.ac.jp
}

\section{ABSTRACT}

Spherical-harmonics expansion method is proposed to solve the quantum time-evolution equations for density matrices numerically in momentum space. This method facilitates efficient real-time simulations of quantum electron dynamics in continuum states through extension of our previous formalism developed for discrete states. Numerical accuracy and efficiency are demonstrated through two examples: (i) multi-photon ionization of a one-electron atom in intense laser field, and (ii) real-time dynamics of plasma oscillations in electron liquids. In case (i), coupled dynamics of density matrices for bound and continuum states reveals an enhancement of multiphoton ionization over the Keldysh approximation through resonant intermediate states.

\section{Introduction}

Quantum dynamics of electrons plays essential roles in nonequilibrium phenomena arising from interactions of atoms, molecules, or condensed matter with time-varying external fields. For instance, femtosecond dynamics of ionization, relaxation, and screening in matter irradiated by $x$-ray free-electron laser is a subject of crucial importance to interpret nonlinear $x$-ray phenomena observed recently [1,2].

In the previous paper [3], the author developed a density-matrix formulation of the time-dependent Hartree-Fock equations for electron systems under a strong external field which is switched on in an arbitrary time scale. With the knowledge of the energies and wave functions of one-electron states $\{k\}$ in the initial unperturbed state, subsequent time evolution of the system after an onset of the external field can be simulated with the one-electron density matrix $\left\langle\rho_{k k^{\prime}}(t)\right\rangle \equiv\left\langle\Psi(t)\left|c_{k}^{\dagger} c_{k^{\prime}}\right| \Psi(t)\right\rangle$. Here, $c_{k}^{\dagger}$ and $c_{k}$ are the creation and annihilation operators, respectively, for state $k$, and $\Psi(\mathrm{t})$ denotes the many-body wave function at time $t$. Preliminary simulations demonstrated a variety of phenomena such as quantum oscillations induced by nonadiabatic perturbations, nonlinear screening of electron gas around an impurity, and Anderson's authogonalization theorem [3]; these simulations were, however, carried out for confined electron systems with discrete energy levels.

Electrons in continuum states, such as the photoelectrons produced through atomic or molecular ionization, Bloch electrons in a crystal, and so forth, are characterized by continuous wave vector k. The simplest way to simulate density matrices for continuum states would be to discretize the $k$-space into finite grid points [4]. Although such a conventional scheme may work for semiconductor Bloch equations [5-8], it may not be applicable to metals, since a sufficiently dense $k$-point mesh is required in order to describe an electronic excitation across a sharp Fermi surface precisely. Moreover, the density matrix related to such an excitation process takes the form $\left\langle\rho_{\mathbf{k}, \mathbf{k}+\mathbf{q}}\right\rangle$, where both $\mathbf{k}$ and $\mathbf{q}$ are (three-dimensional [3D]) continuous vectors $[9,10]$; simultaneous discretization in $\mathbf{k}-$ 
and q-space exhausts too much computer memories.

In this paper, we propose a sphericalharmonics expansion (SHE) method to solve the density-matrix equations numerically for electron systems where transitions among continuum states are dominant. The basic idea is to express the anisotropy of the density matrix in $k$-space in the form of a multipole expansion using the spherical harmonics $Y_{\text {Im }}(\theta, \varphi)$, where the summation over $\ell$ is truncated at some finite value $\left(=\ell_{\max }\right)$. Previous applications of the SHE method were limited to semiclassical dynamics, such as the Boltzmann transport $[11,12]$ or Vlasov simulations [13]. In the present work, we develop the SHE method for fully quantum simulations and examine its accuracy through representative examples. Specifically, we study the multiphoton ionization of a hydrogen-like atom under an intense laser field [14-17], and real-time dynamics of plasma oscillations in electron liquids within the random-phase approximation (RPA) $[9,10]$.

\section{Multiphoton Ionization of an Atom}

We consider the ionization of a $1 \mathrm{~s}$ electron in a hydrogen-like atom by an intense, linearly polarized laser electric field $\boldsymbol{E}(t)=\boldsymbol{E}_{0} \cos (\omega t)$ switched on at time $t=0$. Dominant mechanisms of ionization may be classified [1416] with the Keldysh parameter $\gamma \equiv \sqrt{\left|\varepsilon_{1 s}\right| / 2 U_{p}}$, where $\varepsilon_{1 s}$ denotes the energy level of the $1 \mathrm{~s}$ state, $U_{\mathrm{p}}=e^{2} E_{0}^{2} / 4 m_{\mathrm{e}} \omega^{2}$ refers to the ponderomotive energy, $m_{\mathrm{e}}$ denotes the electron mass, and $e$ is the elemental charge: The regime $\gamma>1$ is dominated by the multiphoton ionization, whereas the tunneling ionization prevails for $\gamma<<1$.

A unified formula of ionization rates that covers the entire $\gamma$-regime was presented by Keldysh [14]. The Keldysh approximation is based on the time-dependent perturbation theory of the transition rates from bound to continuum states by using the Gordon-Volkov wave functions for the latter [17]. While compact analytic formulae for time-averaged ionization rates (including their dependence on the photoelectron momentum) in the Keldysh approximation are available $[16,17]$, transient time evolutions of the ionization process after an onset of the laser field can be explicitly simulated on the basis of the SHE method, as we shall indicate later in this section. We also perform simulations beyond the Keldysh approximation by taking into account $2 \mathrm{~s}$ and $2 \mathrm{p}$ states, to find that these intermediate states enhance the ionization rates significantly.

\section{Basic equations}

General dynamic equations for one-electron density matrices in the time-dependent Hartree-Fock approximation were derived in Ref. [3]. To solve these equations for a hydrogenlike atom, we adopt the exact one-electron energies $\varepsilon_{C}$ and wave functions $\psi_{C}(\mathbf{r})$ for the bound states $c\left(=1 s, 2 s, 2 p_{x, y, z}\right.$, etc.) [18], whereas we assume the free-electron energy spectrum $\varepsilon_{\mathbf{k}}=\hbar^{2} k^{2} / 2 m_{\mathrm{e}}$ and the plane wave $\psi_{\mathbf{k}}(\mathbf{r})=e^{i \mathbf{k} \cdot \mathbf{r}} / \sqrt{\Omega}$ for the continuum, where $\Omega$ denotes the volume of the system. As a consequence, the Coulomb interaction between the ejected electron and the remaining $1 \mathrm{~s}$ hole is neglected as in the original assumption by Keldysh [14]. The dynamics of the density matrix then obeys the following equations

$$
\begin{gathered}
\frac{\partial f_{\mathbf{k}}(t)}{\partial t}=-\frac{2}{\hbar} \operatorname{Im} \sum_{c} \mathbf{d}_{\mathbf{k} c} \cdot \mathbf{E}(t)\left\langle\rho_{\mathbf{k} c}(t)\right\rangle \\
+\frac{e \mathbf{E}(t)}{\hbar} \cdot \frac{\partial f_{\mathbf{k}}(t)}{\partial \mathbf{k}}, \\
\frac{\partial\left\langle\rho_{\mathbf{k} c}(t)\right\rangle}{\partial t}=\frac{i}{\hbar}\left(\varepsilon_{\mathbf{k}}-\varepsilon_{c}\right)\left\langle\rho_{\mathbf{k} c}(t)\right\rangle \\
+\frac{i}{\hbar} \mathbf{d}_{c \mathbf{k}} \cdot \mathbf{E}(t)\left[f_{\mathbf{k}}(t)-f_{c}(t)\right]
\end{gathered}
$$




$$
\begin{aligned}
& +\frac{e \mathbf{E}(t)}{\hbar} \cdot \frac{\partial\left\langle\rho_{\mathbf{k} c}(t)\right\rangle}{\partial \mathbf{k}} \\
& +\frac{i}{\hbar} \mathbf{E}(t) \cdot \sum_{c^{\prime}(\neq c)}\left[\mathbf{d}_{c c^{\prime}}\left\langle\rho_{\mathbf{k} c^{\prime}}(t)\right\rangle-\mathbf{d}_{c^{\prime} \mathbf{k}}\left\langle\rho_{c^{\prime} c}(t)\right\rangle\right] \\
& +\frac{i}{\hbar} \sum_{\mathbf{k}^{\prime}(\neq \mathbf{k})} \mathbf{d}_{c \mathbf{k}^{\prime}} \cdot \mathbf{E}(t)\left\langle\rho_{\mathbf{k} \mathbf{k}^{\prime}}(t)\right\rangle \\
& \frac{\partial f_{c}(t)}{\partial t}=-\frac{2}{\hbar} \mathbf{E}(t) \cdot \operatorname{Im}\left[\sum_{\left.c^{\prime} \neq c\right)} \mathbf{d}_{c c^{\prime}}\left\langle\rho_{c c^{\prime}}(t)\right\rangle\right. \\
& \left.+\sum_{\mathbf{k}} \mathbf{d}_{c \mathbf{k}}\left\langle\rho_{c \mathbf{k}}(t)\right\rangle\right] \\
& \frac{\partial\left\langle\rho_{c c^{\prime}}(t)\right\rangle}{\partial t}=\frac{i}{\hbar}\left(\varepsilon_{c}-\varepsilon_{c^{\prime}}\right)\left\langle\rho_{c c^{\prime}}(t)\right\rangle \\
& +\frac{i}{\hbar} \mathbf{d}_{c^{\prime} c} \cdot \mathbf{E}(t)\left[f_{c}(t)-f_{c^{\prime}}(t)\right] \\
& +\frac{i}{\hbar} \mathbf{E}(t) \cdot \sum_{\mathbf{k}}\left[\mathbf{d}_{c^{\prime} \mathbf{k}}\left\langle\rho_{c \mathbf{k}}(t)\right\rangle-\mathbf{d}_{\mathbf{k} c}\left\langle\rho_{\mathbf{k} c^{\prime}}(t)\right\rangle\right] \\
& +\frac{i}{\hbar} \mathbf{E}(t) \cdot \sum_{c^{\prime \prime}\left(\neq c, c^{\prime}\right)}\left[\mathbf{d}_{c^{\prime} c^{\prime \prime}}\left\langle\rho_{c c^{\prime \prime}}(t)\right\rangle-\mathbf{d}_{c^{\prime \prime} c}\left\langle\rho_{c^{\prime \prime} c^{\prime}}(t)\right\rangle\right], \\
& \text { for } c \neq c^{\prime} \text {, } \\
& \frac{\partial\left\langle\rho_{\mathbf{k k}^{\prime}}(t)\right\rangle}{\partial t}=\frac{i}{\hbar}\left(\varepsilon_{\mathbf{k}}-\varepsilon_{\mathbf{k}^{\prime}}\right)\left\langle\rho_{\mathbf{k k}^{\prime}}(t)\right\rangle \\
& +\frac{e \mathbf{E}(t)}{\hbar} \cdot\left[\frac{\partial\left\langle\rho_{\mathbf{k k}^{\prime}}(t)\right\rangle}{\partial \mathbf{k}^{\prime}}+\frac{\partial\left\langle\rho_{\mathbf{k k}^{\prime}}(t)\right\rangle}{\partial \mathbf{k}}\right] \\
& +\frac{i}{\hbar} \mathbf{E}(t) \cdot \sum_{c}\left[\mathbf{d}_{\mathbf{k}^{\prime} c}\left\langle\rho_{\mathbf{k} c}(t)\right\rangle-\mathbf{d}_{c \mathbf{k}}\left\langle\rho_{c \mathbf{k}^{\prime}}(t)\right\rangle\right]
\end{aligned}
$$

Here, the diagonal component of the density matrix is denoted as $f_{k}(t) \equiv\left\langle\rho_{k k}(t)\right\rangle$, which gives the population of state $k$, and

$$
\begin{aligned}
\mathbf{d}_{c \mathbf{k}} & \equiv \int d \mathbf{r} \psi_{c}^{*}(\mathbf{r})(-e) \mathbf{r} \psi_{\mathbf{k}}(\mathbf{r}), \\
\mathbf{d}_{c c^{\prime}} & \equiv \int d \mathbf{r} \psi_{c}^{*}(\mathbf{r})(-e) \mathbf{r} \psi_{c^{\prime}}(\mathbf{r}),
\end{aligned}
$$

$$
\begin{gathered}
\mathbf{d}_{\mathbf{k} \mathbf{k}^{\prime}} \equiv \int d \mathbf{r} \psi_{\mathbf{k}}^{*}(\mathbf{r})(-e) \mathbf{r} \psi_{\mathbf{k}^{\prime}}(\mathbf{r}) \\
=i e \frac{(2 \pi)^{3}}{\Omega} \frac{\partial \delta\left(\mathbf{k}^{\prime}-\mathbf{k}\right)}{\partial \mathbf{k}^{\prime}}
\end{gathered}
$$

represent the dipole-transition matrix elements.

On the right-hand side of Eq. (1b), we note that the second term accounts for the usual single-photon ionization, while the third kderivative term accounts for the multiphoton ionization [3]; the latter can be derived from the nonlinear coupling term $\sum_{\mathbf{k}^{\prime}(\neq \mathbf{k})} \mathbf{d}_{\mathbf{k}^{\prime} \mathbf{k}}\left\langle\rho_{\mathbf{k}^{\prime} c}(t)\right\rangle$ in the original density-matrix equation [3] with the help of Eq. (2c). The fourth term represents the effect of intermediate bound states on ionization. The final term plays an important role when ionization begins to saturate.

The last term on the right-hand side of Eq. (1a), representing the drift motion of an electron under the electric field, can likewise be derived from the nonlinear coupling term $\sum_{\mathbf{k}^{\prime}(\neq \mathbf{k})} \mathbf{d}_{\mathbf{k k}^{\prime}}\left\langle\rho_{\mathbf{k k}^{\prime}}(t)\right\rangle$; the drift term does not alter the total ionization rate [3] but affects the electron energy distribution in the continuum. We note that the k-derivative terms analogous to those in (1a) and (1b) are relevant also to the dynamical Franz-Keldysh effects in semiconductor quantum wells in intense terahertz electric fields [6].

The density-matrix analysis of atomic multiphoton ionization was put forth earlier by Dixit and Lambropoulos [9] to compute angular distributions of photoelectrons. In their theory, the continuum wave functions were treated rigorously, whereas $\left\langle\rho_{\mathbf{k} c}(t)\right\rangle$ was solved analytically in an approximate way, and $\left\langle\rho_{\mathbf{k k}^{\prime}}(t)\right\rangle$ was not treated explicitly; they regarded the continuum as a sink for the loss of bound-electron populations. In this work, the whole coupled equations (1a)-(1e) are solved numerically.

\section{The spherical-harmonics expansion method}

We now solve Eqs. (1a)-(1e) through the SHE method. We consider a spherical coordinate 
system where $z$-axis is chosen in the direction of $\mathbf{E}$. The direction of $\mathbf{k}$ is then specified by the angles $\left\{\theta_{k}, \varphi_{k}\right\}$ which we shall hereafter abbreviate as $\Omega_{k}$. The diagonal and offdiagonal components of the density matrix are expanded in spherical harmonics as

$$
\begin{aligned}
& f_{\mathbf{k}}(t)=\frac{a_{\mathrm{B}}^{3}}{\Omega} \sum_{\ell=0}^{\ell_{\max }} \sum_{m=-\ell}^{\ell}\left\langle f_{k}^{(\ell m)}(t)\right\rangle_{\Omega} Y_{\ell m}\left(\Omega_{k}\right), \\
& \left\langle\rho_{\mathbf{k} c}(t)\right\rangle=\sqrt{\frac{a_{\mathrm{B}}^{3}}{\Omega} \sum_{\ell=0}^{\ell_{\max }} \sum_{m=-\ell}^{\ell}\left\langle\rho_{k c}^{(\ell m)}(t)\right\rangle_{\Omega} Y_{\ell m}\left(\Omega_{k}\right) .}
\end{aligned}
$$

Here, $a_{\mathrm{B}} \equiv \hbar^{2} / m_{\mathrm{e}} e^{2}$ is the Bohr radius. By repeating a similar procedure to $\mathbf{k}^{\prime}$, we have

$$
\begin{gathered}
\left\langle\rho_{\mathbf{k k}^{\prime}}(t)\right\rangle=\frac{a_{\mathrm{B}}^{3}}{\Omega} \sum_{\ell=0}^{\ell_{\max }} \sum_{m=-\ell}^{\ell} \sum_{\ell^{\prime}=0}^{\ell_{\max }} \sum_{m^{\prime}=-\ell^{\prime}}^{\ell^{\prime}}\left\langle\rho_{k k^{\prime}}^{\left(\ell m, \ell^{\prime} m^{\prime}\right)}(t)\right\rangle_{\Omega} \\
\times Y_{\ell m}\left(\Omega_{k}\right) Y_{\ell^{\prime} m^{\prime}}\left(\Omega_{k^{\prime}}\right) .
\end{gathered}
$$

Since the system under consideration possesses an axial symmetry around $z$-axis, only the $m=0$ components contribute; the SHE reduces to the Legendre-polynomial expansion. We consider only $1 \mathrm{~s}, 2 \mathrm{~s}$ and $2 \mathrm{p}_{\mathrm{z}}$ states, since $2 p_{x}$ and $2 p_{y}$ states do not couple with the ground state through electric dipole transitions. When we substitute (3a)-(3c) into (1a)-(1e) and perform angular integrations, the resultant equations for the expansion coefficients depend only on the magnitude $k \equiv|\mathbf{k}|$ :

$$
\begin{gathered}
\frac{\partial\left\langle f_{k}^{(\ell 0)}(t)\right\rangle_{\Omega}}{\partial t}=\cos (\omega t) \times \\
\left\{-2 \sum_{n=1,2} \Omega_{n s, k} \sum_{\ell^{\prime}=\ell \pm 1} J_{\ell \ell^{\prime}}^{(1)} \operatorname{Re}\left\langle\rho_{k, n s}^{\left(\ell^{\prime} 0\right)}(t)\right\rangle_{\Omega}\right. \\
-2 \Omega_{2 p_{z}, k}^{(2)} \sum_{\ell^{\prime}=\ell, \ell \pm 2} J_{\ell \ell^{\prime}}^{(2)} \operatorname{Im}\left\langle\rho_{k, 2 p_{z}}^{\left(\ell^{\prime} 0\right)}(t)\right\rangle_{\Omega} \\
-2 \Omega_{2 p_{z}, k}^{(0)} \operatorname{Im}\left\langle\rho_{k, 2 p_{z}}^{(\ell 0)}(t)\right\rangle_{\Omega}
\end{gathered}
$$

$$
\begin{gathered}
\left.+\frac{e E_{0}}{\hbar} \sum_{\ell^{\prime}=\ell \pm 1}\left[J_{\ell \ell^{\prime}}^{(1)} \frac{\partial\left\langle f_{k}^{\left(\ell^{\prime} 0\right)}(t)\right\rangle_{\Omega}}{\partial k}+I_{\ell \ell^{\prime}} \frac{\left\langle f_{k}^{\left(\ell^{\prime} 0\right)}(t)\right\rangle_{\Omega}}{k}\right]\right\}^{\prime} \text { (4a) } \\
\frac{\partial\left\langle\rho_{k, 1 s}^{(\ell 0)}(t)\right\rangle_{\Omega}}{\partial t}=\frac{i}{\hbar}\left(\varepsilon_{\mathbf{k}}-\varepsilon_{1 \mathrm{~s}}\right)\left\langle\rho_{k, 1 s}^{(\ell 0)}(t)\right\rangle_{\Omega} \\
+\cos (\omega t)\left\{-\sqrt{\frac{4 \pi}{3}} \delta_{\ell 1^{\prime}} \Omega_{1 s, k} f_{1 s}(t)\right. \\
+\frac{e E_{0}}{\hbar} \sum_{\ell^{\prime}=\ell \pm 1}\left[J_{\ell \ell^{\prime}}^{(1)} \frac{\partial\left\langle\rho_{k, 1 s}^{\left(\ell^{\prime} 0\right)}(t)\right\rangle_{\Omega}}{\partial k}+I_{\ell \ell^{\prime}} \frac{\left\langle\rho_{k, 1 s}^{\left(\ell^{\prime} 0\right)}(t)\right\rangle_{\Omega}}{k}\right]
\end{gathered}
$$$$
+i \Omega_{1 s, 2 \mathrm{p}_{\mathrm{z}}}\left\langle\rho_{k, 2 p_{\mathrm{z}}}^{(\ell 0)}(t)\right\rangle_{\Omega}-\sqrt{\frac{4 \pi}{3}} \delta_{\ell 1} \Omega_{2 s, k}\left\langle\rho_{2 s, 1 \mathrm{~s}}(t)\right\rangle
$$$$
\left.-i\left(\sqrt{\frac{4 \pi}{5}} \delta_{\ell 2} \Omega_{2 p_{z}, k}^{(2)}+\sqrt{4 \pi} \delta_{\ell 0} \Omega_{2 p_{z}, k}^{(0)}\right)\left\langle\rho_{2 p_{z}, 1 \mathrm{~s}}(t)\right\rangle\right\}
$$$$
+\frac{a_{\mathrm{B}}^{3} \cos (\omega t)}{(2 \pi)^{3}} \int_{0}^{\infty} d k^{\prime} k^{\prime 2} \sqrt{\frac{4}{3}} \Omega_{1 s, k^{\prime}}\left\langle\rho_{k k^{\prime}}^{(\ell 0,10)}(t)\right\rangle_{\Omega}{ }^{\prime}
$$$$
\frac{\partial\left\langle\rho_{k, 2 s}^{(\ell 0)}(t)\right\rangle_{\Omega}}{\partial t}=(1 s \leftrightarrow 2 s) \text { in (4b) , }
$$$$
\frac{\partial\left\langle\rho_{k, 2 p_{z}}^{(\ell 0)}(t)\right\rangle_{\Omega}}{\partial t}=\frac{i}{\hbar}\left(\varepsilon_{\mathbf{k}}-\varepsilon_{2 p_{z}}\right)\left\langle\rho_{k, 2 p_{z}}^{(\ell 0)}(t)\right\rangle_{\Omega}
$$$$
+\cos (\omega t)\left\{-i\left(\sqrt{\frac{4 \pi}{5}} \delta_{\ell 2} \Omega_{2 p_{z}, k}^{(2)}+\sqrt{4 \pi} \delta_{\ell 0} \Omega_{2 p_{z}, k}^{(0)}\right) f_{2 p_{z}}(t)\right.
$$$$
+\frac{e E_{0}}{\hbar} \sum_{\ell^{\prime}=\ell \pm 1}\left[J_{\ell \ell^{\prime}}^{(1)} \frac{\partial\left\langle\rho_{k, 2 p_{z}}^{\left(\ell^{\prime} 0\right)}(t)\right\rangle_{\Omega}}{\partial k}+I_{\ell \ell^{\prime}} \frac{\left\langle\rho_{k, 2 p_{z}}^{\left(\ell^{\prime} 0\right)}(t)\right\rangle_{\Omega}}{k}\right]
$$$$
+\sum_{n=1,2}\left[i \Omega_{n s, 2 \mathrm{p}_{z}}\left\langle\rho_{k, n s}^{(\ell 0)}(t)\right\rangle_{\Omega}\right.
$$

$$
\begin{gathered}
\left.\left.-\sqrt{\frac{4 \pi}{3}} \delta_{\ell 1} \Omega_{n s, k}\left\langle\rho_{2 p_{z}, n s}(t)\right\rangle^{*}\right]\right\} \\
+i \frac{a_{\mathrm{B}}^{3} \cos (\omega t)}{(2 \pi)^{3}} \int_{0}^{\infty} d k^{\prime} k^{\prime 2}\left[\sqrt{\frac{4 \pi}{5}} \Omega_{2 p_{z}, k^{\prime}}^{(2)}\left\langle\rho_{k k^{\prime}}^{(\ell 0,20)}(t)\right\rangle_{\Omega}\right. \\
\left.+\sqrt{4 \pi} \Omega_{2 p_{z}, k^{\prime}}^{(0)}\left\langle\rho_{k k^{\prime}}^{(\ell 0,00)}(t)\right\rangle_{\Omega}\right], \quad(4 \mathrm{~d})
\end{gathered}
$$




$$
\begin{aligned}
& \frac{\partial f_{1 s}(t)}{\partial t}=2 \cos (\omega t) \Omega_{1 s, 2 p_{z}} \operatorname{Im}\left\langle\rho_{2 p_{z}, 1 s}(t)\right\rangle \\
& \quad+\frac{a_{\mathrm{B}}^{3} \cos (\omega t)}{\sqrt{12 \pi^{5}}} \int_{0}^{\infty} d k k^{2} \Omega_{1 s, k} \operatorname{Re}\left\langle\rho_{k, 1 s}^{(10)}(t)\right\rangle_{\Omega}, \quad \text { (4e) }
\end{aligned}
$$$$
\frac{\partial f_{2 s}(t)}{\partial t}=(1 s \leftrightarrow 2 s) \text { in }(4 \mathrm{e})
$$

$$
\begin{aligned}
& \frac{\partial f_{2 p_{z}}(t)}{\partial t}=-2 \cos (\omega t) \sum_{n=1,2} \Omega_{n s, 2 p_{z}} \operatorname{Im}\left\langle\rho_{2 p_{z}, n s}(t)\right\rangle \\
&+\frac{a_{\mathrm{B}}^{3} \cos (\omega t)}{4 \pi^{3}} \int_{0}^{\infty} d k k^{2}\left[\sqrt{\frac{4 \pi}{5}} \Omega_{2 p_{z}, k}^{(2)} \operatorname{Im}\left\langle\rho_{k, 2 p_{z}}^{(20)}(t)\right\rangle_{\Omega}\right. \\
&\left.+\sqrt{4 \pi} \Omega_{2 p_{z}, k}^{(0)} \operatorname{Im}\left\langle\rho_{k, 2 p_{z}}^{(00)}(t)\right\rangle_{\Omega}\right], \quad(4 \mathrm{~g})
\end{aligned}
$$$$
\frac{\partial\left\langle\rho_{2 s, 1 \mathrm{~s}}(t)\right\rangle}{\partial t}=\frac{i}{\hbar}\left(\varepsilon_{2 s}-\varepsilon_{1 \mathrm{~s}}\right)\left\langle\rho_{2 s, 1 \mathrm{~s}}(t)\right\rangle
$$$$
+\frac{a_{\mathrm{B}}^{3} \cos (\omega t)}{(2 \pi)^{3}} \int_{0}^{\infty} d k k^{2} \sqrt{\frac{4 \pi}{3}}\left[\Omega_{1 s, k}\left\langle\rho_{k, 2 s}^{(10)}(t)\right\rangle_{\Omega}^{*}\right.
$$$$
\left.+\Omega_{2 s, k}\left\langle\rho_{k, 1 s}^{(10)}(t)\right\rangle_{\Omega}\right]
$$$$
+i \cos (\omega t)\left[\Omega_{1 s, 2 p_{z}}\left\langle\rho_{2 p_{z}, 2 s}(t)\right\rangle^{*}-\Omega_{2 s, 2 p_{z}}\left\langle\rho_{2 p_{z}, 1 s}(t)\right\rangle\right],
$$

$$
\begin{aligned}
& \frac{\partial\left\langle\rho_{2 p_{z}, 1 \mathrm{~s}}(t)\right\rangle}{\partial t}=\frac{i}{\hbar}\left(\varepsilon_{2 p_{z}}-\varepsilon_{1 \mathrm{~s}}\right)\left\langle\rho_{2 p_{z}, 1 \mathrm{~s}}(t)\right\rangle \\
& -i \cos (\omega t) \Omega_{1 s, 2 p_{z}}\left[f_{1 s}(t)-f_{2 p_{z}}(t)\right] \\
& +\frac{a_{\mathrm{B}}^{3} \cos (\omega t)}{(2 \pi)^{3}} \int_{0}^{\infty} d k k^{2}\left[\Omega_{1 s, k} \sqrt{\frac{4 \pi}{3}}\left\langle\rho_{k, 2 p_{z}}^{(10)}(t)\right\rangle_{\Omega}^{*}\right. \\
& \left.-i \sqrt{\frac{4 \pi}{5}} \Omega_{2 p_{z}, k}^{(2)}\left\langle\rho_{k, 1 s}^{(20)}(t)\right\rangle_{\Omega}-i \sqrt{4 \pi} \Omega_{2 p_{z}, k}^{(0)}\left\langle\rho_{k, 1 s}^{(00)}(t)\right\rangle_{\Omega}\right] \\
& -i \cos (\omega t) \Omega_{2 s, 2 p_{z}}\left\langle\rho_{2 s, 1 s}(t)\right\rangle, \\
& \frac{\partial\left\langle\rho_{2 p_{z}, 2 \mathrm{~s}}(t)\right\rangle}{\partial t}=(1 s \leftrightarrow 2 s) \text { in }(4 \mathrm{i}),
\end{aligned}
$$

$$
\begin{aligned}
& \frac{\partial\left\langle\rho_{k k^{\prime}}^{\left(\ell 0, \ell^{\prime} 0\right)}(t)\right\rangle_{\Omega}}{\partial t}=\frac{i}{\hbar}\left(\varepsilon_{\mathbf{k}}-\varepsilon_{\mathbf{k}^{\prime}}\right)\left\langle\rho_{k k^{\prime}}^{\left(\ell 0, \ell^{\prime} 0\right)}(t)\right\rangle_{\Omega} \\
& +\frac{e E_{0} \cos (\omega t)}{\hbar} \times \\
& \left\{\sum_{\ell^{\prime \prime}=\ell^{\prime} \pm 1}\left[J_{\ell^{\prime} \ell^{\prime \prime}}^{(1)} \frac{\partial\left\langle\rho_{k k^{\prime}}^{\left(\ell 0, \ell^{\prime \prime} 0\right)}(t)\right\rangle}{\partial k^{\prime}}+I_{\ell^{\prime} \ell^{\prime \prime}} \frac{\left\langle\rho_{k k^{\prime}}^{\left(\ell, \ell^{\prime \prime} 0\right)}(t)\right\rangle}{k^{\prime}}\right]\right. \\
& \left.+\sum_{\ell^{\prime \prime}=\ell \pm 1}\left[J_{\ell \ell^{\prime \prime}}^{(1)} \frac{\partial\left\langle\rho_{k k^{\prime}}^{\left(\ell^{\prime \prime} 0, \ell^{\prime} 0\right)}(t)\right\rangle}{\partial k}+I_{\ell \ell^{\prime \prime}} \frac{\left\langle\rho_{k k^{\prime}}^{\left(\ell^{\prime \prime} 0, \ell^{\prime} 0\right)}(t)\right\rangle}{k}\right]\right\} \\
& -\cos (\omega t) \sqrt{\frac{4 \pi}{3}} \sum_{n=1,2}\left[\delta_{\ell^{\prime} 1} \Omega_{n s, k^{\prime}}\left\langle\rho_{k, n s}^{(\ell 0)}(t)\right\rangle_{\Omega}\right. \\
& \left.-\delta_{\ell 1} \Omega_{n s, k}\left\langle\rho_{k^{\prime}, n s}^{\left(\ell^{\prime} 0\right)}(t)\right\rangle_{\Omega}^{*}\right] \\
& +i \cos (\omega t) \times \\
& {\left[\left(\sqrt{\frac{4 \pi}{5}} \delta_{\ell^{\prime} 2} \Omega_{2 p_{z}, k^{\prime}}^{(2)}+\sqrt{4 \pi} \delta_{\ell^{\prime} 0} \Omega_{2 p_{z}, k^{\prime}}^{(0)}\right)\left\langle\rho_{k, 2 p_{z}}^{(\ell 0)}(t)\right\rangle_{\Omega}\right.} \\
& \left.-\left(\sqrt{\frac{4 \pi}{5}} \delta_{\ell 2} \Omega_{2 p_{z}, k}^{(2)}+\sqrt{4 \pi} \delta_{\ell 0} \Omega_{2 p_{z}, k}^{(0)}\right)\left\langle\rho_{k^{\prime}, 2 p_{z}}^{\left(\ell^{\prime} 0\right)}(t)\right\rangle_{\Omega}^{*}\right] .
\end{aligned}
$$

Here,

$$
\begin{gathered}
\Omega_{1 s, k}=32 \sqrt{\pi} e a_{\mathrm{B}} \frac{Z^{5 / 2} k a_{\mathrm{B}}}{\left[\left(k a_{\mathrm{B}}\right)^{2}+Z^{2}\right]^{3}} \frac{E_{0}}{\hbar}, \\
\Omega_{2 s, k}=16 \sqrt{2 \pi} e a_{\mathrm{B}} \frac{Z^{5 / 2} k a_{\mathrm{B}}\left[\left(k a_{\mathrm{B}}\right)^{2}-\frac{Z^{2}}{4}\right]}{\left[\left(k a_{\mathrm{B}}\right)^{2}+\frac{Z^{2}}{4}\right]^{4}} \frac{E_{0}}{\hbar}, \\
\Omega_{2 p_{z}, k}^{(2)}=\sqrt{8 \pi} e a_{\mathrm{B}} \frac{4 Z^{7 / 2}\left(k a_{\mathrm{B}}\right)^{2}}{\left[\left(k a_{\mathrm{B}}\right)^{2}+\frac{Z^{2}}{4}\right]^{4}} \frac{E_{0}}{\hbar}, \\
\Omega_{2 p_{z}, k}^{(0)}=\sqrt{8 \pi} e a_{\mathrm{B}} \frac{Z^{7 / 2}\left[\left(k a_{\mathrm{B}}\right)^{2}-\frac{Z^{2}}{4}\right]}{\left[\left(k a_{\mathrm{B}}\right)^{2}+\frac{Z^{2}}{4}\right]^{4}} \frac{E_{0}}{\hbar},
\end{gathered}
$$




$$
\begin{aligned}
& \Omega_{1 s, 2 p_{z}}=-\frac{128 \sqrt{2}}{243} \frac{e a_{\mathrm{B}}}{Z} \frac{E_{0}}{\hbar} . \\
& \Omega_{2 s, 2 p_{z}}=3 \frac{e a_{\mathrm{B}}}{Z} \frac{E_{0}}{\hbar}
\end{aligned}
$$

refer to the characteristic (Rabi) frequencies and $\delta_{\ell \ell^{\prime}}$ denotes Kronecker's delta. In Eqs. (4a-4k), we have used the fact that $f_{c}(t) \gg f_{\mathbf{k}}(t)$ when the system volume $\Omega$ is macroscopically large.

The coefficients $I_{r \ell}$ and $J_{r \ell}^{(n)}$ that enter Eqs. (4) are defined as

$$
\begin{gathered}
I_{r \ell} \equiv \int d \Omega_{k} Y_{r 0}^{*}\left(\Omega_{k}\right)\left(1-\cos ^{2} \theta_{k}\right) \frac{\partial Y_{\ell 0}\left(\Omega_{k}\right)}{\partial \cos \theta_{k}}, \\
J_{r \ell}^{(n)} \equiv \int d \Omega_{k} Y_{r 0}^{*}\left(\Omega_{k}\right) P_{n}\left(\cos \theta_{k}\right) Y_{\ell 0}\left(\Omega_{k}\right),
\end{gathered}
$$

where $P_{n}\left(\cos \theta_{k}\right)$ denotes the Legendre polynomial. These integrations can be carried out with the aid of Wigner's 3-j symbols [18]; the resultant non-zero components can be expressed compactly as follows ( $n$ : integer).

$$
\begin{gathered}
I_{2 n, 2 n-1}=-\frac{2 n(2 n-1)}{\sqrt{(4 n+1)(4 n-1)}}, \\
I_{2 n, 2 n+1}=-\frac{2}{3} \frac{n}{4 n-1} \frac{40 n^{2}+18 n-7}{\sqrt{(4 n+1)(4 n+3)}} \\
+\frac{2}{3} \sqrt{(4 n+1)(4 n+3)}, \\
I_{2 n+1,2 n}=-\frac{2 n(2 n+1)}{\sqrt{(4 n+3)(4 n+1)}}, \\
I_{2 n+1,2 n+2}=-\frac{2}{3} \frac{2 n+1}{4 n+1} \frac{20 n^{2}+29 n+6}{\sqrt{(4 n+3)(4 n+5)}} \\
J_{\ell, \ell-1}^{(1)}=\frac{\frac{2}{3} \sqrt{(4 n+3)(4 n+5)},}{\sqrt{(2 \ell+1)(2 \ell-1)}},
\end{gathered}
$$

$$
\begin{gathered}
J_{\ell, \ell+1}^{(1)}=\frac{\ell+1}{\sqrt{(2 \ell+1)(2 \ell+3)}}, \\
J_{\ell, \ell-2}^{(2)}=\frac{3}{2} \frac{1}{\sqrt{(2 \ell+1)(2 \ell-3)}} \frac{\ell(\ell-1)}{2 \ell-1}, \\
J_{\ell, \ell}^{(2)}=\frac{(\ell+1) \ell}{(2 \ell+3)(2 \ell-1)}, \\
J_{\ell, \ell+2}^{(2)}=\frac{3}{2} \frac{1}{\sqrt{(2 \ell+5)(2 \ell+1)}} \frac{(\ell+2)(\ell+1)}{2 \ell+3} .
\end{gathered}
$$

We note that $J_{r \ell}^{(n)}$ is often denoted as $c^{n}(r 0, \ell 0)$ in atomic physics [18].

The set of coupled Eqs. (4a)-(4k) for $\ell, \ell^{\prime}=0,1, \cdots, \ell_{\max }$ are integrated numerically with the initial condition $\left\langle f_{1 s}(0)\right\rangle=1$ (all the other matrix elements are zero at $t=0$ ), using the staggered leap-frog algorithm with a time step $\Delta t$. The variables $k, k^{\prime}\left(0 \leq k, k^{\prime} \leq k_{\max }\right)$ are divided equally into $N_{k}$ discrete points. We have adopted $\ell_{\max }=24-32, k_{\max }=3 \mathrm{au}, N_{k}=$ 300-400, and $\Delta t=0.0025$ au in our simulations.

\section{Simulations in the Keldysh approximation}

We have computed time evolutions of the multiphoton ionization processes of a hydrogen atom $(Z=1)$ for photon energy $\hbar \omega=5 \mathrm{eV}$. To examine the performance of the SHE method, we first perform simulations within the Keldysh approximation. The Keldysh approximation [14] is equivalent to making the following simplifications: (i) The contributions from $2 \mathrm{~s}$ and $2 p_{z}$ states are neglected. We thus retain Eqs. (4a) and (4b) but neglect (4c)-(4k). We likewise retain (5a) while (5b)-(5f) are set equal to zero. (ii) On the right-hand side of Eq. (4b), the last term containing the $k^{\prime}$-integration is ignored. (iii) We set $f_{1 s}(t) \approx 1$ on the right-hand side of Eq. (4b) to ignore saturation effects.

The total ionization rate at time $t$ can be calculated in accordance with $w(t)=\partial f_{\text {cont }}(t) / \partial t$, where 


$$
\begin{aligned}
f_{\text {cont }}(t) & \equiv \sum_{\mathbf{k}} f_{\mathbf{k}}(t) \\
& =\frac{a_{\mathrm{B}}^{3}}{4 \pi^{5 / 2}} \int_{0}^{k_{\max }} d k k^{2}\left\langle f_{k}^{(00)}(t)\right\rangle_{\Omega}
\end{aligned}
$$

corresponds to the continuum population. In Figure 1, we display the time-averaged ionization rates,

$$
\bar{w}(t) \equiv \frac{1}{t-t_{0}} \int_{t_{0}}^{t} d t^{\prime} w\left(t^{\prime}\right)
$$

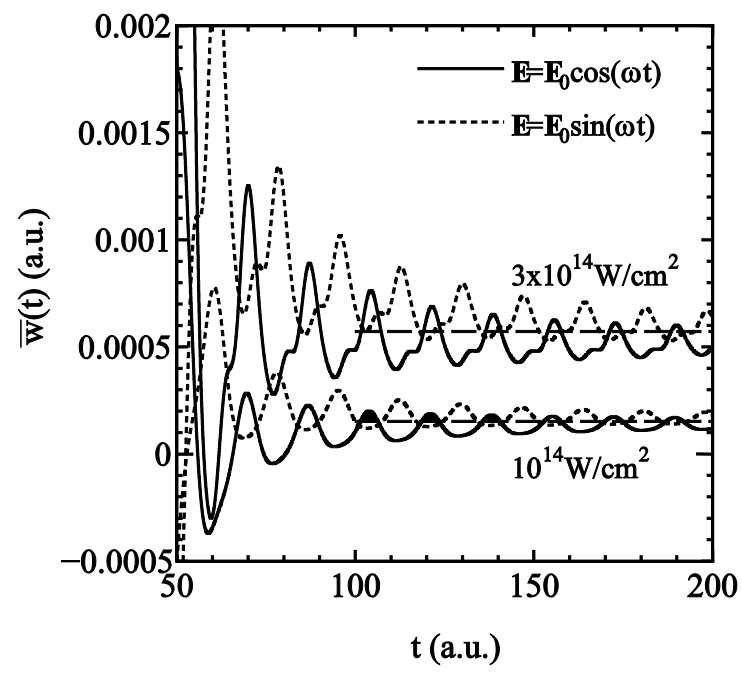

Figure 1. Time-averaged ionization rates (10) (solid and dotted curves) for multiphoton ionization of a hydrogen atom with $I_{v}=10^{14}$ $\mathrm{W} / \mathrm{cm}^{2}$ and $3 \times 10^{14} \mathrm{~W} / \mathrm{cm}^{2}$, compared with the Keldysh ionization rates in the asymptotic limit [14] (dashed curves). The photon energy is $5 \mathrm{eV}$ in all the cases.

Here, we adopt $t_{0}=50$ au in order to remove the initial transient variations of $w(t)$. Numerical results are indicated by the solid curves. We consider two cases with different laser intensities $I_{v} \equiv(c / 8 \pi) E_{0}^{2}=10^{14} \mathrm{~W} / \mathrm{cm}^{2}$ $(\gamma=3.4)$ and $3 \times 10^{14} \mathrm{~W} / \mathrm{cm}^{2} \quad(\gamma=2.0)$; the minimum number of absorbed photons is 3 and 4, respectively. For comparison, we have performed analogous simulations for the case
$E(t)=E_{0} \sin (\omega t)$, which are shown by the dotted curves. Although $\bar{w}(t)$ oscillates and depend on the initial phase of the electric field, the phase-averaged values for large $t$ turn out to be consistent with the asymptotic Keldysh rates [14]. In the previous paper [3], we have indeed proven that analytic solutions to (1a) and (1b) in a moving frame reproduce the Keldysh ionization rates [14] in the limit $t \rightarrow \infty$.

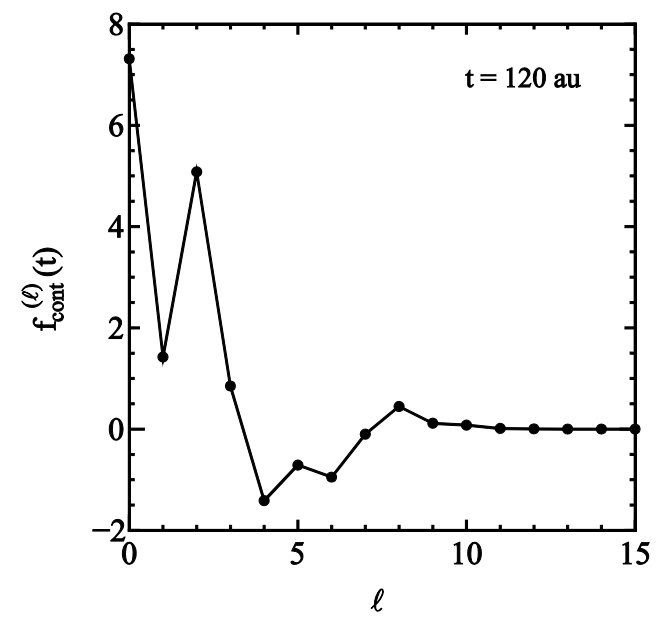

Figure 2. Convergence of $f_{\text {cont }}^{(\ell)}(t)$ with respect to $\ell$ at $t=120 \mathrm{au}$; the laser intensity is $I_{v}=2.42 \times 10^{14} \mathrm{~W} / \mathrm{cm}^{2}$.

To assess the convergence of the SHE, we decompose the continuum population into multipole contributions according to

$$
f_{\text {cont }}^{(\ell)}(t) \equiv a_{\mathrm{B}}^{3} \int_{0}^{k_{\max }} d k k^{2}\left\langle f_{k}^{(\ell 0)}(t)\right\rangle_{\Omega} .
$$

Numerical results at $t=120$ au are shown in Figure 2. It can be seen that the contributions from $\ell<10$ are dominant. The cutoff $\ell_{\text {max }}=32$ adopted in this work is thus large enough to warrant numerical accuracy, yet much more efficient than the two-dimensional discretization in the cylindrical coordinate because $\ell_{\max }<<N_{k}$. 


\section{Simulations including $2 s$ and $2 p$ states}

We proceed to the full simulations of Eqs. (4a)(4k) for a hydrogen atom to see how the intermediate $2 \mathrm{~s}$ and $2 \mathrm{p}_{\mathrm{z}}$ states affect the dynamics of multiphoton ionization. Since $\varepsilon_{2 s}-\varepsilon_{1 s}=\varepsilon_{2 p_{z}}-\varepsilon_{1 s}=10.20 \mathrm{eV}$, these levels are located close to the two-photon resonance for $\hbar \omega=5 \mathrm{eV}$. The simulation parameters in this subsection are chosen as $\ell_{\text {max }}=24$ and $N_{k}=$ 300.

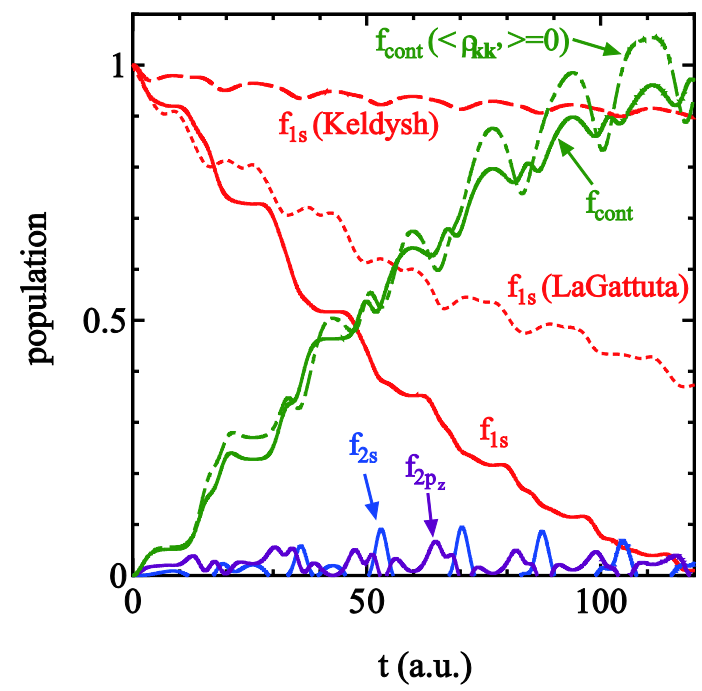

Figure 3. Time evolutions of populations in the multiphoton ionization of a hydrogen atom for $\hbar \omega=5 \mathrm{eV}$ and $I_{v}=2.42 \times 10^{14} \mathrm{~W} / \mathrm{cm}^{2}$. The solid curves depict our full simulations. The dotdashed curve indicates $f_{\text {cont }}(t)$ obtained by setting $\left\langle\rho_{\mathbf{k k}^{\prime}}(t)\right\rangle \equiv 0$ in (1b). The dashed curve represents $f_{1 s}(t)$ simulated in the Keldysh approximation; the dotted curve is the corresponding result by LaGattuta [20].

Figure 3 displays the time evolutions of populations for bound levels and continuum. A comparison between the full simulation and the Keldysh approximation reveals that the resonant $2 s$ and $2 p_{z}$ states tend to enhance the ionization significantly, although the populations of these states remain lower than about 0.1. These features are qualitatively in accord with the more rigorous calculation by LaGattuta [20] based on the direct numerical solutions to the time-dependent Schrödinger equation. Quantitatively, our simulations predict somewhat larger ionization rates compared with LaGattuta's data, as shown by the rapid decrease of $f_{1 s}$ in Figure 3.

Our simulations show that $f_{\text {cont }}(t)$ eventually saturates and approaches unity. When we carry out analogous simulations by intentionally neglecting the last term on the right-hand side of Eq. (1b) related to $\left\langle\rho_{\mathbf{k k}^{\prime}}(t)\right\rangle$, an unphysical feature $f_{\text {cont }}(t)>1$ appears for $t>$ 100 au as indicated by the dot-dashed curve in Figure 3. Thus, the coupling between $\left\langle\rho_{\mathbf{k} c}(t)\right\rangle$ and $\left\langle\rho_{\mathbf{k k}^{\prime}}(t)\right\rangle$ plays an important role in ionization dynamics at high intensities.

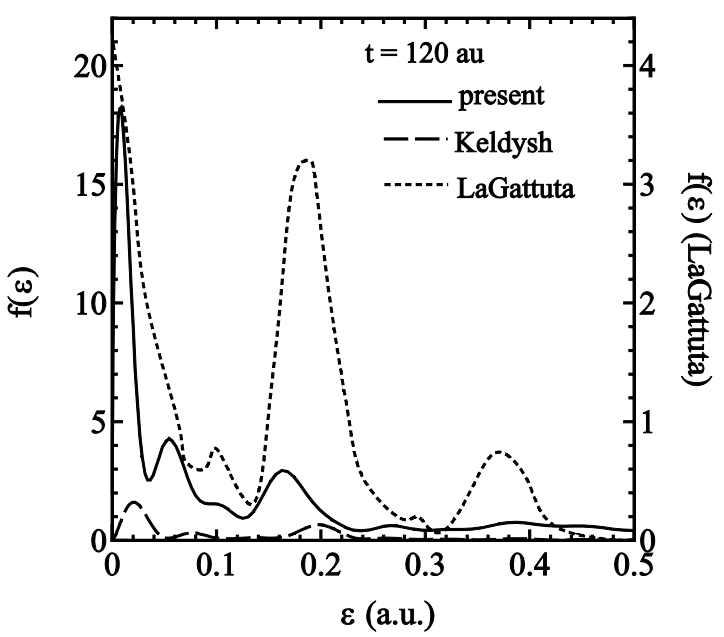

Figure 4. Photoelectron energy distributions at $t=120$ au for $\hbar \omega=5 \mathrm{eV}$ and $I_{v}=2.42 \times 10^{14}$ $\mathrm{W} / \mathrm{cm}^{2}$. The solid and dashed curves depict Eq. (12) computed with the full simulation and the simulation in the Keldysh approximation, respectively. The dotted curve represents the simulation result by LaGattuta [20] with the scale given on the right axis.

Transient photoelectron energy spectrum at time $t$ can be evaluated in accordance with 
$f(\varepsilon, t)=\frac{1}{4 \pi^{5 / 2}} \frac{k a_{\mathrm{B}}^{2}}{e^{2}}\left\langle f_{k}^{(00)}(t)\right\rangle_{\Omega}, k=\frac{\sqrt{2 m_{\mathrm{e}} \varepsilon}}{\hbar}$.

This function satisfies the normalization $\int_{0}^{\infty} d \varepsilon f(\varepsilon, t)=f_{\text {cont }}(t)$. The spectra so computed for $I_{v}=2.42 \times 10^{14} \mathrm{~W} / \mathrm{cm}^{2}$ and $t=120$ au are shown in Figure 4 and compared with the calculation by LaGattuta [20]. The highest peak at 0.009 au and a smaller peak at 0.16 au in the full simulation may be interpreted as the threeand four-photon peak, respectively, which appear in LaGattuta's data [20] as well. The energy of the four-photon peak, however, turns out to be somewhat lower than LaGattuta's prediction. Moreover, the height of the threephoton peak in this work is significantly larger than that of the four-photon peak, whereas the two peak heights obtained by LaGattuta are roughly comparable.

A possible origin of the discrepancies between the present and LaGattuta's data in Figures 3 and 4 might be ascribed to the treatment of continuum wave functions. As we have used plane waves, the resultant boundfree dipole transition matrix elements (5a)-(5d) correspond to those in the Born approximation. It has been known that the Born approximation generally breaks down at energies near ionization threshold. There are a variety of methods to obtain improved continuum wave functions near threshold, such as the distortedwave approximation [21], iterative Schwinger method [22], and B-spline method [23]. The wave functions obtained through these methods may be used to construct more accurate transition matrix elements that replace (5a)-(5d). These are the issues to be studied in the future.

We have also found that the neglect of the $\left\langle\rho_{\mathbf{k k}^{\prime}}(t)\right\rangle$-related term in (1b) results in an unphysical feature $f(\varepsilon, t)<0$ for certain ranges of $\varepsilon$, which again indicates an importance of the coupling between (1b) and (1e).

\section{Plasma Oscillations in Electron Liquids}

Plasma oscillation is a collective motion of electrons mutually interacting via Coulomb forces $[9,10]$. It has been investigated intensively in particular for an electron liquid in a uniform background of positive compensating charges $[9,10]$. Let us define $\omega_{q}$ as the frequency of the plasma oscillation (or plasmon) with wave vector $q$, and introduce a dimensionless frequency $v_{q} \equiv \hbar \omega_{q} / 2 \varepsilon_{\mathrm{F}}$, where $\varepsilon_{\mathrm{F}}=\hbar^{2} k_{\mathrm{F}}^{2} / 2 m_{\mathrm{e}}$ is the Fermi energy, $k_{\mathrm{F}}=\left(3 \pi^{2} n_{\mathrm{e}}\right)^{1 / 3}$ is the Fermi wave number, and $n_{\mathrm{e}}$ is the electron number density. In the meanfield RPA theory, the dispersion relation in the long-wavelength regime is known to be $[9,10,24]$

$$
v_{q}^{2}=v_{\mathrm{p}}^{2}+\frac{3}{5}\left(\frac{q}{k_{\mathrm{F}}}\right)^{2}+O\left(\left(\frac{q}{k_{\mathrm{F}}}\right)^{4}\right) .
$$

Here, the plasma frequency is given by $\omega_{\mathrm{p}}=\left(4 \pi n_{\mathrm{e}} e^{2} / m_{\mathrm{e}}\right)^{1 / 2}$, and $v_{\mathrm{p}} \equiv \hbar \omega_{\mathrm{p}} / 2 \varepsilon_{\mathrm{F}}$ is its dimensionless form. For shorter wavelengths, damping of plasmon occurs through resonant particle-hole excitation $[9,10,24]$, where quantum dynamics plays an essential role.

The plasmon dispersion can be directly obtained through measurements or calculations of the dynamic structure factor $S(q, \omega)$, which carries information on the density-fluctuation spectrum $[9,10,25]$. It has been therefore customary to analyze plasma oscillations in frequency domain. In ultrashort laser-pulse experiments, however, plasma oscillations may be suddenly excited by a strong perturbation applied to the electron system. A real-time analysis of plasma oscillation has been scarce so far, except for a linear-response analysis by Allen [26] or semiclassical approximations $[13,27]$. In this section, we shall perform fully quantum simulations of plasma oscillations with the SHE method.

\section{Basic equations}


Let us apply the general dynamical equations for the one-electron density matrix presented in Ref. [3] to a paramagnetic electron liquid in the ground state. We introduce a notation $\left\langle\rho_{\mathbf{k}}(\mathbf{q}, t)\right\rangle \equiv\left\langle\rho_{\mathbf{k}, \mathbf{k}+\mathbf{q}}(t)\right\rangle \quad$ which manifests a process of an electron-hole pair excitation with momentum $-\mathbf{q}$ (see Figure 5 ). The one-electron energies and wave functions are given as $\varepsilon_{\mathbf{k}}=\hbar^{2} k^{2} / 2 m_{\mathrm{e}} \quad$ and $\quad \psi_{\mathbf{k}}(\mathbf{r})=e^{i \mathbf{k} \cdot \mathbf{r}} / \sqrt{\Omega} \quad$ ， respectively, where the exchange self-energy [28] has been neglected. The resultant densitymatrix equations read

$$
\begin{gathered}
\frac{\partial f_{\mathbf{k}}(t)}{\partial t}=\frac{2}{\hbar} \operatorname{Im} \sum_{\mathbf{q}(\neq 0)} \tilde{\varepsilon}_{\mathbf{k}, \mathbf{k}+\mathbf{q}}(t)\left\langle\rho_{\mathbf{k}}(\mathbf{q}, t)\right\rangle, \\
\frac{\partial\left\langle\rho_{\mathbf{k}}(\mathbf{q}, t)\right\rangle}{\partial t}=\frac{i}{\hbar}\left(\varepsilon_{\mathbf{k}}-\varepsilon_{\mathbf{k}+\mathbf{q}}\right)\left\langle\rho_{\mathbf{k}}(\mathbf{q}, t)\right\rangle \\
-\frac{i}{\hbar} \tilde{\varepsilon}_{\mathbf{k}+\mathbf{q}, \mathbf{k}}(t)\left[f_{\mathbf{k}}(t)-f_{\mathbf{k}+\mathbf{q}}(t)\right] .
\end{gathered}
$$

The Hartree interaction among electrons responsible for the plasma oscillation is taken into account through the self-energy matrix [3]

$$
\tilde{\varepsilon}_{\mathbf{k}+\mathbf{q}, \mathbf{k}}=\frac{2 v(q)}{\Omega}\left\langle\rho_{\mathbf{q}}(t)\right\rangle=\tilde{\varepsilon}_{\mathbf{k}, \mathbf{k}+\mathbf{q}}^{*}
$$

with $v(q) \equiv 4 \pi e^{2} / q^{2}$. Note that Eq. (15) is independent of $\mathbf{k}$. Our primary concern is the Fourier component of the density fluctuation

$$
\left\langle\rho_{\mathbf{q}}(t)\right\rangle \equiv \sum_{\mathbf{k}}\left\langle\rho_{\mathbf{k}}(\mathbf{q}, t)\right\rangle
$$

This quantity is related to the local electron density at position $\mathbf{r}$ via the Fourier transform $n_{\mathrm{e}}(\mathbf{r})=(2 / \Omega) \sum_{\mathbf{q}}\left\langle\rho_{\mathbf{q}}(t)\right\rangle e^{i \mathbf{q} \cdot \mathbf{r}}$. Formulae (14a) and (14b) constitute the time-evolution equations equivalent to RPA.

We suppose that a small electron-hole pair excitation $\left\langle\rho_{\mathbf{k}}(\mathbf{q}, 0)\right\rangle$ preexists at $t=0$ and figure out whether it triggers spontaneous plasma oscillations [26]. Specifically, $\left\langle\rho_{\mathbf{k}}(\mathbf{q}, 0)\right\rangle$ is assumed to take the form

$$
\left\langle\rho_{\mathbf{k}}(\mathbf{q}, 0)\right\rangle=\frac{v_{\mathrm{ext}}(q)}{\Omega} \frac{f_{\mathbf{k}}(0)-f_{\mathbf{k}+\mathbf{q}}(0)}{\varepsilon_{\mathbf{k}}-\varepsilon_{\mathbf{k}+\mathbf{q}}+i \hbar \Gamma}
$$

where $\Gamma$ is a positive infinitesimal, and

$$
f_{\mathbf{k}}(0)=\left\{\begin{array}{ll}
1, & k \leq k_{\mathrm{F}} \\
0, & k>k_{\mathrm{F}}
\end{array} .\right.
$$

Equation (17) corresponds to the density matrix induced by a weak, hypothetical local potential $v_{\text {ext }}(q)$ in noninteracting electron gas, that can be derived through the first-order perturbation theory [3].

\section{The spherical-harmonics expansion method}

To solve Eqs. (14a) and (14b), we fix the vector $\mathbf{q}$ and adopt SHE for $\mathbf{k}$ as

$$
\begin{aligned}
& f_{\mathbf{k}}(t)=\sum_{\ell=0}^{\ell_{\max }} \sum_{m=-\ell}^{\ell} f_{k}^{(\ell m)}(t) Y_{\ell m}\left(\Omega_{k}\right) \\
& \left\langle\rho_{\mathbf{k}}(\mathbf{q}, t)\right\rangle=\frac{a_{\mathrm{B}}^{3} \sum_{\max }^{\ell}}{\Omega} \sum_{\ell=0}^{\ell}\left\langle\rho_{m=-\ell}^{(\ell m)}(\mathbf{q}, t)\right\rangle_{\Omega} Y_{\ell m}\left(\Omega_{k q}\right) \cdot
\end{aligned}
$$

Here, $\Omega_{k} \equiv\left\{\theta_{k}, \varphi_{k}\right\}$ specifies the direction of $\mathbf{k}$ in $x y z$-frame fixed in the laboratory, whereas $\Omega_{k q} \equiv\left\{\theta_{k q}, \varphi_{k q}\right\} \quad$ denotes the corresponding angle in $X Y Z$-frame which is obtained by rotating the $x y z$-frame in such a way that the $Z$ axis points to the direction of $\mathbf{q}$.

To simplify the problem, we consider a case where $v_{\text {ext }}(q)$ is spherically symmetric. Since the Fermi surface is also spherical, we may set $f_{\mathbf{k}}(t)=f_{k}^{(00)}(t) / 2 \sqrt{\pi}$ in (19a). Equation (14a) then reduces to

$$
\begin{aligned}
& \frac{\partial f_{k}^{(00)}(t)}{\partial t} \\
& =\frac{4}{\hbar} \operatorname{Im} \frac{1}{(2 \pi)^{3}} \frac{a_{\mathrm{B}}^{3}}{\Omega} \int d \mathbf{q} v(q)\left\langle\rho_{-\mathbf{q}}(t)\right\rangle\left\langle\rho_{k}^{(00)}(\mathbf{q}, t)\right\rangle_{\Omega} \\
& \rightarrow 0(\Omega \rightarrow \infty) .
\end{aligned}
$$


Here, the right-hand side contains the system volume $\Omega$ in the denominator and hence vanishes for a macroscopic system $\Omega \rightarrow \infty$. Thus, $f_{k}^{(00)}(t)$ is independent of $t$, which means that the initial Fermi distribution (18) remains unchanged: $f_{k}^{(00)}=2 \sqrt{\pi}$ for $k \leq k_{\mathrm{F}} \quad$ and $f_{k}^{(00)}=0$ for $k>k_{\mathrm{F}}$.

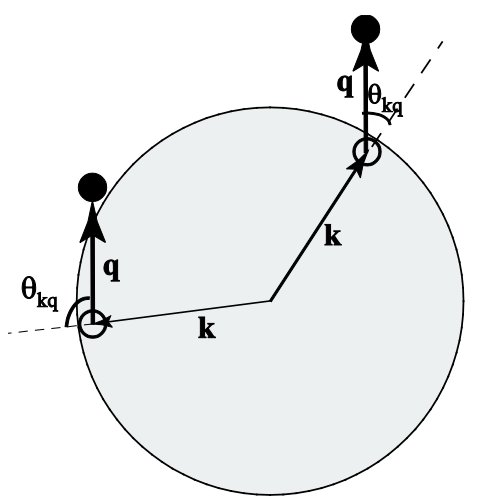

Figure 5. Anisotropic nature of the electronhole excitation processes in the Fermi sphere. The two processes indicated by the same excitation wave vector $\mathbf{q}$ are inequivalent, depending on the relative angle $\theta_{k q}$ between $\mathbf{k}$ and $\mathbf{q}$.

Because the system is axially symmetric in k-space once $\mathbf{q}$ is fixed, only the $m=0$ components remain in equation (19b). We emphasize, however, that the summation over $\ell$ must be fully retained, because the probability of electron-hole excitation depends on the direction of $\mathbf{k}$ for a given $\mathbf{q}$, as illustrated in Figure 5. Noting that $\varepsilon_{\mathbf{k}+\mathbf{q}}$ can be expressed in terms of $\cos \theta_{k q}$, equation (14b) can be rewritten as

$$
\begin{aligned}
& \frac{\partial\left\langle\rho_{k}^{(\ell))}(\mathbf{q}, t)\right\rangle_{\Omega}}{\partial t} \\
& =-i \frac{\hbar k q}{m_{\mathrm{e}}}\left[J_{\ell, \ell-1}^{(1)}\left\langle\rho_{k}^{(\ell-1,0)}(\mathbf{q}, t)\right\rangle_{\Omega}+J_{\ell, \ell+1}^{(1)}\left\langle\rho_{k}^{(\ell+1,0)}(\mathbf{q}, t)\right\rangle_{\Omega}\right] \\
& -\frac{i \hbar q^{2}}{2 m_{\mathrm{e}}}\left\langle\rho_{k}^{(\ell 0)}(\mathbf{q}, t)\right\rangle_{\Omega} \\
& -i \frac{v(q)}{\hbar a_{\mathrm{B}}^{3}} \frac{\sqrt{2 \ell+1}}{k q}\left\langle\rho_{\mathbf{q}}(t)\right\rangle \\
& \quad \times \int_{|k-q|}^{k+q} d k^{\prime} k^{\prime} P_{\ell}\left(\cos \theta_{k q}\right)\left(f_{k}^{(00)}-f_{k^{\prime}}^{(00)}\right), \quad(21)
\end{aligned}
$$

where $\cos \theta_{k q} \equiv\left(k^{\prime 2}-k^{2}-q^{2}\right) / 2 k q ; J_{\ell, \ell-1}^{(1)}$ and $J_{\ell, \ell+1}^{(1)}$ are given by (8e) and (8f), respectively. By combining equation (16) with (19b), the density fluctuation $\left\langle\rho_{\mathbf{q}}(t)\right\rangle$ that enters equation (21) can be expressed as

$$
\left\langle\rho_{\mathbf{q}}(t)\right\rangle=\frac{a_{\mathrm{B}}^{3}}{4 \pi^{5 / 2}} \int_{0}^{k_{\max }} d k k^{2}\left\langle\rho_{k}^{(00)}(\mathbf{q}, t)\right\rangle_{\Omega} .
$$

We remark that the volume factor $\Omega$ does not appear explicitly in equation (21) unlike equation (20).

The initial condition (17) can likewise be rewritten in the SHE form. We assume a Yukawa potential for $v_{\text {ext }}(q)$ and adopt the form

$$
\begin{aligned}
\left\langle\rho_{k}^{(\ell, 0)}(q, 0)\right\rangle_{\Omega}= & -\left(\frac{2 \pi}{k_{\mathrm{F}}}\right)^{3} \frac{A}{\left(\frac{q}{k_{\mathrm{F}}}\right)^{2}+B} \frac{\sqrt{2 \ell+1}}{2 k q} \\
& \times \int_{|k-q|}^{k+q} d k^{\prime} k^{\prime} P_{\ell}\left(\cos \theta_{k q}\right) \\
& \times \frac{f_{k}^{(00)}-f_{k^{\prime}}^{(00)}}{\left(k a_{\mathrm{B}}\right)^{2}-\left(k^{\prime} a_{\mathrm{B}}\right)^{2}+i \Gamma^{\prime}},
\end{aligned}
$$

where we choose the (arbitrary) model parameters as $A=0.01, B=1$, and $\Gamma^{\prime}=10^{-8}$. We remark that equation (23) is independent of the directions of $\mathbf{q}$, and so is equation (21). 


\section{Results}

Equation (21) for $\ell=0,1, \cdots, \ell_{\max }$ has been integrated numerically with initial condition (23) for electron density $r_{\mathrm{s}} \equiv\left(3 / 4 \pi n_{\mathrm{e}}\right)^{1 / 3} / a_{\mathrm{B}}=4$. The midpoint algorithm has been employed. The variable $k$ $\left(0 \leq k \leq k_{\max }\right)$ has been divided into $N_{k}$ grid points; the $k^{\prime}$-integrations in (21) and (23) can also be evaluated as discrete summations over these grid points. The simulation parameters are $\ell_{\max }=24, k_{\max }=2.5 k_{\mathrm{F}}, N_{k}=400$, and the time step $\Delta t=0.01 \hbar / 2 \varepsilon_{\mathrm{F}}$. We have also performed simulations with $k_{\max }=2.0 k_{\mathrm{F}}$ to find that the results are virtually the same.

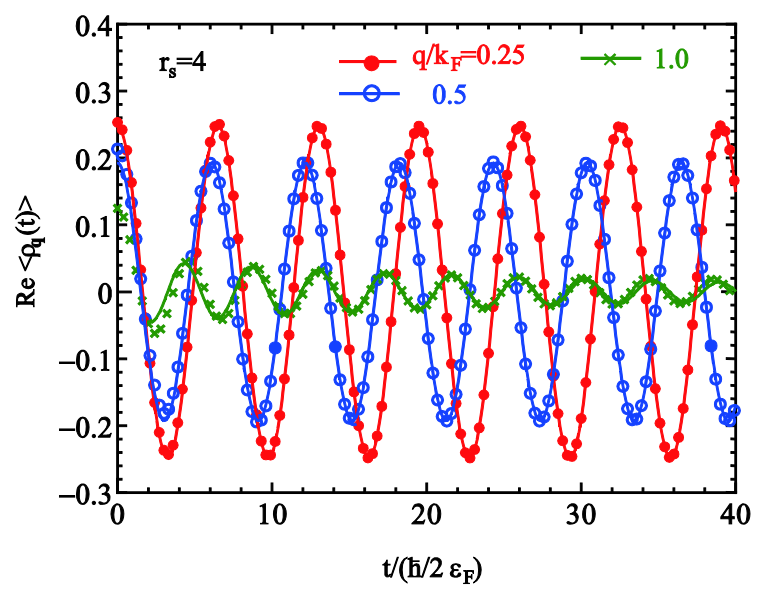

Figure 6. Real part of the density fluctuation (22) for $r_{s}=4$. The filled circles, empty circles and crosses are the computed data for $q / k_{\mathrm{F}}=$ $0.25,0.5$, and 1.0 , respectively; the solid curves are the corresponding fits.

Figure 6 indicates time evolutions of $\operatorname{Re}\left\langle\rho_{\mathbf{q}}(t)\right\rangle$ for various values of $q$, which clearly manifest collective plasma oscillations. The computed data can be fitted with the functional form $\operatorname{Re}\left\langle\rho_{\mathbf{q}}(t)\right\rangle=C \cos \left(\omega_{q} t\right) \exp \left(-\gamma_{q} t\right)$, where $\omega_{q}$ gives the frequency of the plasmon, and $\gamma_{q}$ is the corresponding damping factor. It can be observed that $\omega_{q}$ is an increasing function of $q$, indicating a positive dispersion relation. For $q / k_{\mathrm{F}}=0.25$ and 0.5 , the damping factor can be neglected $\left(\gamma_{q}=0\right)$, while the data for $q / k_{\mathrm{F}}=1.0$ exhibit a small damping $\hbar \gamma_{q} / 2 \varepsilon_{\mathrm{F}} \approx 0.03$.

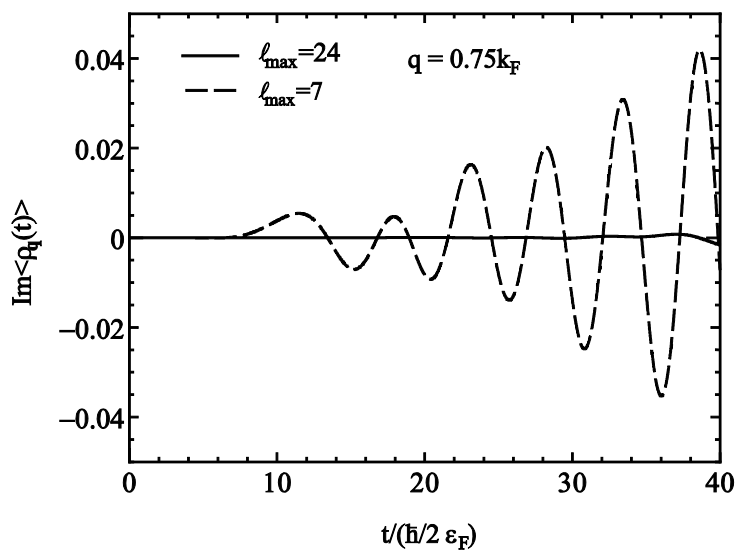

Figure 7. Imaginary part of the density fluctuation (22) for $r_{\mathrm{s}}=4$ and $q=0.75 k_{\mathrm{F}}$. The solid and dashed curves correspond to the case with $\ell_{\max }=24$ and 7, respectively.

By definition, the density fluctuation (16) should satisfy the relation $\left\langle\rho_{-\mathbf{q}}(t)\right\rangle=\left\langle\rho_{\mathbf{q}}(t)\right\rangle^{*}$. Because $\left\langle\rho_{\mathbf{q}}(t)\right\rangle$ depends only on the magnitude of $\mathbf{q}$ in the numerical examples treated in this work, it follows that $\operatorname{Im}\left\langle\rho_{\mathbf{q}}(t)\right\rangle=0$; this criterion can be used to judge the numerical accuracy of the simulations. It can be seen in Figure 7 that, when $\ell_{\max }$ is too small, spurious oscillations of $\operatorname{Im}\left\langle\rho_{\mathbf{q}}(t)\right\rangle$ appear, whereas such oscillations can be 
$q / k_{F}=1$ in Figure 6 indeed exhibits a damped oscillation, which is consistent with the RPA theory.

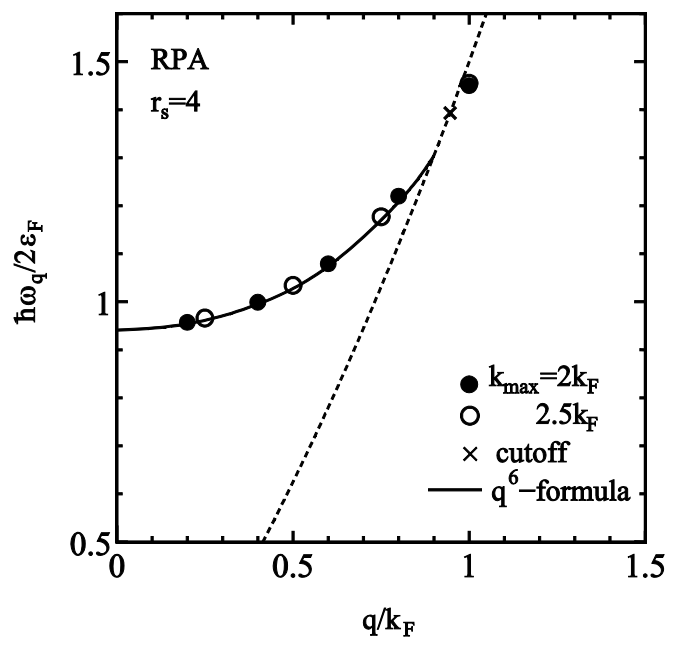

Figure 8. The plasmon dispersion relation for $r_{\mathrm{s}}$ $=4$. Black and white circles indicate numerical results for $k_{\max }=2 k_{\mathrm{F}}$ and $2.5 k_{\mathrm{F}}$, respectively. The dotted curve represents the boundary of the particle-hole continuum. The cutoff $q_{c}$ given by Eq. (25) is marked with the cross. The solid curve depicts the analytic RPA formula (24).

\section{Conclusion and Outlook}

We have proposed a SHE method to solve the density-matrix equations of quantum electron dynamics in continuum states. We have thereby shown that this method is applicable to real-time dynamics of both atomic and condensed-matter processes, such as multiphoton ionization of a hydrogen-like atom and plasma oscillations in electron liquids. In both cases, numerical accuracy has been achieved by setting $\ell_{\text {max }} \approx 20-30$ : These values are only slightly larger than the typical values for semi-classical Boltzmann equations [11,12] $\left(\ell_{\max } \approx 1-20\right)$ and hence an efficiency of the SHE

which yields $q_{\mathrm{c}}=0.946 k_{\mathrm{F}}$ for $r_{\mathrm{s}}=4$, as indicated
by the cross in Figure 8 . The plasmon damping should occur for $q>q_{c}$; our numerical result for 
has been ensured for quantum simulations as well.

The main achievement in the present work is to solve fully coupled dynamics of density matrices for both bound and continuum states. Specifically, we have demonstrated a significant enhancement of multiphoton ionization due to resonant $2 \mathrm{~s}$ and $2 \mathrm{p}$ states. The boundcontinuum density matrix $\left\langle\rho_{\mathbf{k}, 1 s}(t)\right\rangle$ is directly responsible for the ionization of a 1 s electron, which in turn is coupled with the continuum density matrix $\left\langle\rho_{\mathbf{k k}^{\prime}}(t)\right\rangle$; the latter quantity plays a central role in the dynamics of plasma oscillation in an electron liquid.

The final goal of the present SHE method is to simulate ultrafast quantum phenomena in which both localized and delocalized electrons participate. As a relevant example, we mention a solid or cluster irradiated by an x-ray freeelectron laser pulse, in which localized core holes are created through photoionization and delocalized valence electrons respond to them.

The present simulation of atomic photoionization has been limited to a oneelectron atom. Photoionization of a multielectron atom can in principle be formulated by incorporating the self-energy matrices that account for electron-electron or electron-hole interactions in excited states [3]. It remains to be seen whether the spindependent self-energy matrix in the unrestricted formalism [3] can describe correlated motions of ionizing and remaining electrons [29] without invoking the conventional single active electron approximation [15].

For a molecule or a crystal, the bound-free transition matrix element (2a) may be generalized as $\mathbf{d}_{c \mathbf{k}}(\mathbf{R}) \equiv \int d \mathbf{r} \psi_{c}^{*}(\mathbf{r}-\mathbf{R})(-e) \mathbf{r} \psi_{\mathbf{k}}(\mathbf{r})$ for an atom located at $\mathbf{R}$. In the case of a crystal, the relation $\mathbf{d}_{c \mathbf{k}}(\mathbf{R})=e^{i \mathbf{k} \cdot \mathbf{R}} \mathbf{d}_{c \mathbf{k}}(0)$ holds due to the Bloch theorem, producing an additional factor $e^{i \mathbf{k} \cdot \mathbf{R}}$ that has to be handled. When a simple axial symmetry is broken, the transformation of a spherical harmonics between laboratory and molecular frame would have to be used extensively [30]. Plasma oscillations induced by general random potentials are no longer isotropic so that the expansion (19a) should be carried out explicitly. Further developments would be necessary along these lines.

\section{Acknowledgments}

This work was supported in part through Grantin-Aid for Scientific Research (A) provided by JSPS.

Keywords: quantum electron dynamics, density matrix, spherical-harmonics expansion, multiphoton ionization, plasma oscillation

\section{References and Notes}

1. H. Yoneda, Y. Inubushi, M. Yabashi, T. Katayama, T. Ishikawa, H. Ohashi, H. Yumoto, K. Yamauchi, H. Mimura, H. Kitamura, Nat. Comm. 2014, 5, 5080.

2. N. Rohringer, D. Ryan, R.A. London, M. Purvis, F. Albert, J. Dunn, J.D.Bozek, C. Bostedt, A. Graf, R. Hill, S.P. Hau-Riege, J.J. Rocca, Nature 2012, 481, 488.

3. H. Kitamura, Int. J. Quant. Chem. 2014, 114, 1518.

4. D.W. Bailey, J.M. Higman, J. Comp. Phys. 1995, 120, 117.

5. F. Rossi, T. Kuhn, Rev. Mod. Phys. 2002, 74, 895.

6. C.J. Dent, B.N. Murdin, I. Galbraith, Phys. Rev. B 2003, 67, 165312. 
7. M. Hirtschulz, F. Milde, E. Malic, C. Thomsen, S. Reich, A. Knorr, Phys. Stat. Sol. (b) 2008, 245, 2164.

8. O. Hess, T. Kuhn, Phys. Rev. A 1996, 54, 3360 .

9. S. Ichimaru, Rev. Mod. Phys. 1982, 54, 1017.

10. D. Pines, P. Nozieres, The Theory of Quantum Liquids; Perseus Books: MS, Section 5.2, 1999.

11. B. Jacob, P.N. Robson, P.R. David, G.J. Rees, J. Appl. Phys. 2002, 91, 5438.

12. C. Jungemann, A.T. Pham, B. Meinerzhagen, C. Ringhofer, $M$. Bollhöfer, J. Appl. Phys. 2006, 100, 024502.

13. A. Dellafiore, F. Matera, F.A. Brieva, Phys. Rev. B 2000, 61, 2316.

14. L.V. Keldysh, Sov. Phys. JETP 1965, 20, 1307.

15. M. Protopapas, C.H. Keitel, P.L. Knight, Rep. Prog. Phys. 1997, 60, 389.

16. V.S. Popov, Phys. Uspekhi 2004, 47, 855.

17. K. Nagaya, K. Mishima, M. Hayashi, S.H. Lin, J. Phys. Soc. Jpn 2006, 75, 044302.

18. R.D. Cowan, The Theory of Atomic Structure and Spectra; Univ. of California Press: Berkeley, Chapters 3 and 5, 1981.

19. D.N. Dixit, P. Lambropoulos, Phys. Rev. A 1983, 27, 861.
20. K.J. LaGattuta, Phys. Rev. A 1990, 41, 5110.

21. D.G. Arbó, J.E. Miraglia, M.S. Gravielle, K. Schiessl, E. Persson, J. Burgdörfer, Phys. Rev. A 2008, 77, 013401.

22. R.R. Lucchese, G. Raseev, V. McKoy, Phys. Rev. A 1982, 25, 2572.

23. H. Bachau, E. Cormier, P. Decleva, J.E. Hansen, F. Martín, Pep. Prog. Rhys. 2001, 64, 1815.

24. R.A. Ferrell, Phys. Rev. 1957, 107, 450.

25. W. Schülke, Electron Dynamics by Inelastic X-Ray Scattering; Oxford Univ. Press: NY, Chapter 2, 2007.

26. P.B. Allen, From Quantum Mechanics to Technology, p.125; Z. Petru, J. Przystawa, K. Rapcewicz, Ed.; Springer: Berlin, 1996.

27. G. Manfredi, P.-A. Hervieux, Comp. Mater. Sci. 2006, 35, 327.

28. A.L. Fetter, J.D. Walecka, Quantum Theory of Many-Particle Systems, Chapter 4; McGraw-Hill: NY, 1971.

29. J. Caillat, J. Zanghellini, M. Kitzler, O. Koch, W. Kreuzer, A. Scrinzi, Phys. Rev. A 2005, 71, 012712.

30. A.R. Edmonds, Angular Momentum in Quantum Mechanics, Chapter 4; Princeton Univ. Press: NJ, 1974. 